\title{
Frequency-dependency Characterization of Electromagnetic Reverberation in Indoor Scenarios Based on 1-40 GHz Channel Measurements
}

\author{
Marwan Yusuf, Emmeric Tanghe, Maria-Teresa Martinez-Ingles, Juan Pascual-Garcia, \\ Jose-Maria Molina-Garcia-Pardo, Luc Martens, and Wout Joseph
}

\begin{abstract}
A novel model for the reverberation time is proposed in this letter, based on Room Electromagnetics theory. While the commonly used models consider absorption based on a constant rate of wall interactions within a given time, the proposed model takes the variance of interactions into account. Moreover, the frequency-dependency of electromagnetic reverberation characteristics in indoor environments is experimentally investigated from 1 to $40 \mathrm{GHz}$. The reverberation time is found to be a decreasing function of frequency. A model is then developed to predict the room's quality factor, reverberation time, and average absorption coefficient. Good agreement is obtained with the limited results reported in the literature for similar scenarios. This approach aims to be an accurate alternative to the reverberation time measurements and computations in indoor environments by linking it to the theory of electromagnetic fields in cavities.
\end{abstract}

Index Terms-room electromagnetics, Dense multipath components, indoor modeling, reverberation time, absorption rate

\section{INTRODUCTION}

$\mathbf{S}$ IGNIFICANT attention within wireless networks industry and academia has been given to frequencies above $6 \mathrm{GHz}$. The future $5 \mathrm{G}$ standards will exploit the new spectrum for higher peak data rates and network throughput [1]. Various frequency bands $(24,25-27.5 \mathrm{GHz}, 31.8-33.4 \mathrm{GHz}$ and $37-$ $40.5 \mathrm{GHz}$ bands) have been identified as potential candidates at the last World Radio Conference (WRC) by the International Telecommunication Union (ITU) [2]. Therefore, it is of great importance to characterize the underlying propagation environments across the ultra-wideband (UWB) frequencies using efficient models of low-complexity.

In realistic indoor environments, diffuse scattering is becoming a relevant propagation mechanism, in terms of the contribution of the dense multipath components (DMC) to the total power density [3]. Similar to Room Acoustics [4], Room Electromagnetics theory views the indoor environment as a lossy cavity, where all the effective losses can be described by the exponentially decaying tail of the diffuse fields in time [5]. The decay time constant, also known as Reverberation

This work was funded by the EOS project "Multi-service Wireless Network (MUSE-WINET)"

Marwan Yusuf, Emmeric Tanghe, Luc Martens and Wout Joseph are with the Department of Information Technology, IMEC-WAVES, Ghent University, Belgium (e-mail: marwan.yusuf@ugent.be)

Maria-Teresa Martinez-Ingles, Juan Pascual-Garcia and Jose-Maria MolinaGarcia-Pardo are with Departamento Tecnologías de la Información y las Comunicaciones, Universidad Politécnica de Cartagena, Spain
Time (RT), is a function of the volume and the absorption area of the room [6], [7]. The RT can also be related to the quality factor $(Q)$ of the room. Being regarded as lossy cavities, rooms in commercial and residential buildings generally have low $Q$ values [8]. A general description of the reverberation time is based on Sabine's reverberation theory [9]. It assumes homogeneous repartition of energy within the room, and consequently uniformly distributed absorption, and that the field is completely diffuse. In general, the field will be sufficiently diffuse if the basic dimensions of the room are almost the same, walls are not parallel, and most absorbing surfaces are divided into parts and uniformly distributed. In practice, almost none of these requirements is fulfilled [4]. In the case of a room in which absorption is not uniformly distributed, the reverberation time frequency characteristics cannot be predicted accurately using Sabines or other classical reverberation theories. Different approaches have been adopted to obtain more accurate approximations of the reverberation time. Among others, Eyring presented his remarkable paper that described the reverberation in highly absorbent enclosures based on the mean free path between reflections [10].

The work in [5], [6] validated the use of the acoustic reverberation models of Sabine and Eyring in electromagnetics by observing the RT and power gain, while altering the properties of the room in a controlled manner. It confirmed that the reverberation is confined in the room where the transmitter (Tx) and receiver (Rx) are located. However, these models are derived based on several simplifications. One approximation is that the number of reflections is constant over time. This approximation can be further improved by introducing a variance to the number of reflections at a given time [4]. In addition, the RT has normally been investigated at a single frequency [5], [6], [7]. Whilst the RT can be estimated using computational-based methods [11], excessive processing time and memory resources are needed especially at higher frequencies. The measurement-based method in [12] has been used to address the frequency dependency of the RT in the 2$10 \mathrm{GHz}$ band. It showed that the RT decreases for increasing frequency and validated the model by measurement and by comparison to reported results in the literature. Other work [13] suggested that the indoor scenario ceases to reverberate and the room electromagnetic theory is not applicable anymore at frequencies as high as $94 \mathrm{GHz}$.

The novelty of this letter is based on introducing a modified room electromagnetics model that takes the probability distri- 
bution of the number of reflections into account, instead of only the mean value considered by Eyring [4]. Furthermore, we develop a frequency-dependent model for the reverberation time and the chamber $Q$ for indoor scenarios in the band of 1-40 GHz. The extension to this higher frequency band fills the gap in the previously reported frequency characterization.

The letter is organized as follows. Section II introduces the model for RT and $Q$, as well as the proposed modification to account for the variance in the number of reflections based on the geometry of the room. The measurement scenario and methodology are described in Section III. In Section IV, the frequency-dependent models for $Q$-factor, RT and average absorption coefficient are presented, while conclusions are drawn in Section V.

\section{PROPOSED MODELS}

\section{A. Reverberation model}

The original work of Sabine for room acoustics relies on the assumption of a diffuse field that is homogeneously distributed inside the room [4]. However, Sabine's model is not appropriate for rooms with high absorption rate, as pointed out by Eyring [10]. For low- $Q$ or "dead" rooms, Eyring proposed another model that is based on the mirror source theory. For cavities, the received energy is the incoherent summation of rays from infinitely many mirror sources representing multiple wall reflections [4]. Energy received from each mirror source is reduced by the absorption coefficient $\alpha$ when crossing a boundary of an image room. Therefore, the average energy density $W(t)$ received at time $t$ is approximately equal to [6]

$$
W(t)=\frac{E_{0}}{V}(1-\alpha)^{n t}=\frac{E_{0}}{V} \exp (-t / \tau)
$$

where $V$ is the volume of the room, $E_{0}$ is the initial energy generated by mirror sources, and $n$ is the average rate of wall interactions or boundary crossings as aforementioned. The assumption of a diffuse field reveals that $n=\frac{c A}{4 V}$ [4]. Hence, the decay rate of the energy density, which defines the RT, can be expressed as

$$
\tau=\frac{-4 V}{c A \ln (1-\alpha)}
$$

where $A$ is the surface area of the room and $c$ is the speed of light. This represents Eyring's formula for the RT in dead rooms.

One of the simplifications (1) is based on is the replacement of the actual number of wall interactions $N$ in a given time by its average $\mathrm{E}[N]=n t$ [4]. This approximation can be improved by introducing the probability $P_{t}(N)$ of exactly $N$ wall interactions occurring in a time $t$ and calculating the energy density as the expectation with respect to this probability distribution. This can be formulated as

$$
W(t)=\frac{E_{0}}{V} \sum_{N=0}^{\infty} P_{t}(N) \exp (-N a)
$$

where the absorption exponent $a=-\ln (1-\alpha)$ is introduced. Using a series expansion of the exponential term and truncating it after the second order, we reach [4]

$$
W(t)=\frac{E_{0}}{V} \exp (-n t a)\left(1+\frac{1}{2} \sigma_{N}^{2} a^{2}\right)
$$

where $\sigma_{N}^{2}=\sum_{N}(N-n t)^{2} P_{t}(N)$ is the variance of $N$. The number of interactions $N$ in a given time can be related to the actual free path length $l$ traveled between the interactions, whose distribution around the mean $\bar{l}$ and its variance depend on the shape of the room. According to [4], it can be assumed that

$$
\sigma_{N}^{2}=n t \gamma^{2}
$$

where $\gamma^{2}=\frac{\overline{l^{2}}-(\bar{l})^{2}}{(\bar{l})^{2}}$ is the relative variance of the path length $l$. As long as $\frac{1}{2} \sigma_{N}^{2} a^{2}<1$, substituting (5) in (4) approximately yields

$$
W(t)=\frac{E_{0}}{V} \exp \left[-n t a\left(1-\frac{1}{2} \gamma^{2} a\right)\right]
$$

Accordingly, the modified RT can be expressed as

$$
\tau_{m}=\frac{-4 V}{c A \ln (1-\alpha)\left[1+\frac{\gamma^{2}}{2} \ln (1-\alpha)\right]}
$$

\section{B. Q-factor model}

From the theory of electromagnetic fields in cavities, $Q$ factor is defined as the ratio of the energy stored to the energy dissipated in the cavity per cycle of duration $1 / f$ [8]. Alternatively, the decay-time method relates $Q$ to the RT by the following expression [8]

$$
Q=2 \pi f \tau
$$

Since the RT is a function of $V / A$ in (2) and (7), It would be useful to work with a $Q$ density that is independent of the room's dimensions. Hence, the $Q$ per unit (volume per area) can be defined as

$$
Q_{d}=2 \pi f \tau \frac{A}{V}
$$

which describes the frequency-dependency of the RT, as shown in [12].

\section{Measurement Scenario and Methodology}

\section{A. Scenario}

The scenario for the channel sounding measurements is a laboratory in Universidad Politécnica de Cartagena, Spain. The measurement scenario is depicted in Fig. 1 (a), in which Rx is fixed in one position, while Tx positions are uniformly distributed across the room (marked 1 to 14). For all positions, a $0.8 \mathrm{~m}(1.2 \mathrm{~m})$ distance was selected between each Tx row (column). All distances were measured with a laser to obtain the most accurate precision possible. The laboratory size is approximately $9.1 \times 4.8 \times 4.1 \mathrm{~m}^{3}$. It is furnished with several cupboards, chairs, and shelves. The walls are typical interior plasterboard, while the floor and ceiling are made of concrete.

\section{B. Measurement}

In order to perform the channel sounding, a Vector Network Analyzer (VNA) of type Rohde \& Schwarz (R\&S ZVA67 $10 \mathrm{MHz}-67 \mathrm{GHz}$ ) and a radio-over-fiber link (EMCORE Optiva OTS-2, $50 \mathrm{MHz}-40 \mathrm{GHz}$ ) have been used to measure the complex gain of the indoor UWB channel ranging from $1 \mathrm{GHz}$ up to $40 \mathrm{GHz}$. UWB antennas (STEATITE Q-par 


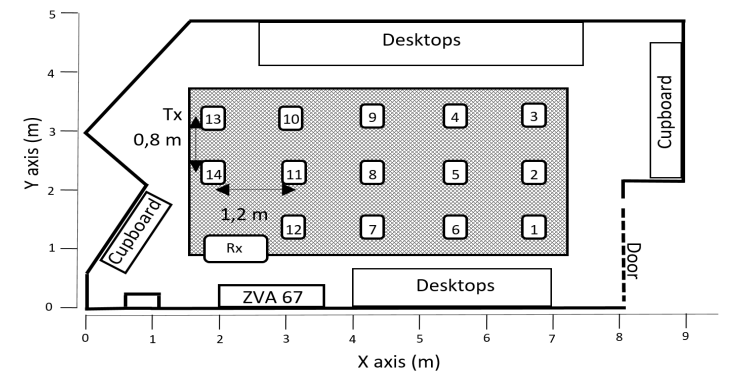

(a)

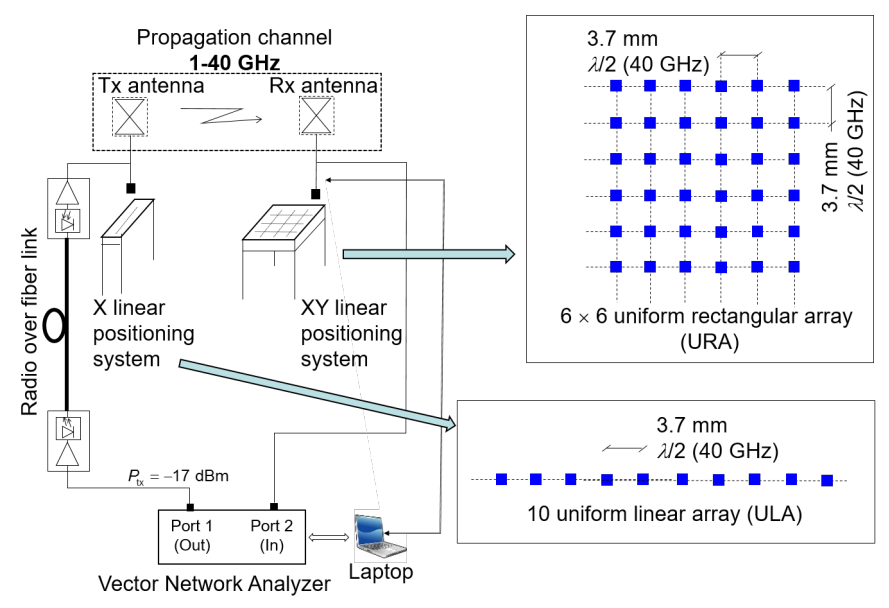

(b)

Fig. 1. (a) Top view of the laboratory used as measurement scenario (b) Measurement setup scheme

Antennas, 0.8-40 GHz) have been used at the Tx and Rx, with vertical polarization and omnidirectional radiation pattern in the horizontal plane. All elements have been calibrated, and the antenna patterns have been measured in an anechoic chamber from 1 to $40 \mathrm{GHz}$ [14]. A scheme of the full measurement setup is shown in Fig. 1 (b).

At both ends of the measurement system, a virtual antenna array was created by an automated positioning system on which the antennas were mounted. This virtual Multiple-Input Multiple-Output (MIMO) measurement system consists of a 10-element Uniform Linear Array (ULA) at the Tx and a $6 \times 6$ Uniform Rectangular Array (URA) at the Rx. Hence, a total of 360 complex transfer functions have been recorded within a duration of around 20 hours for each measured snapshot. During that time, the doors remained closed to prevent entry, thus guaranteeing static conditions. At each position, the VNA measured the complex gain by sampling the $39 \mathrm{GHz}$ span over 8192 uniformly spaced frequency points with intermediate frequency of $100 \mathrm{~Hz}$.

\section{Processing}

According to the theory of room electromagnetics [5], the decay rate of the power density is independent of the position of measurement. The power delay profile (PDP) per Tx position is calculated by averaging the square of the channel impulse response over all the elements of the Tx and $\mathrm{Rx}$ antennas. Since we only care for the slope of the PDP, a more accurate RT can be estimated by spatially averaging the

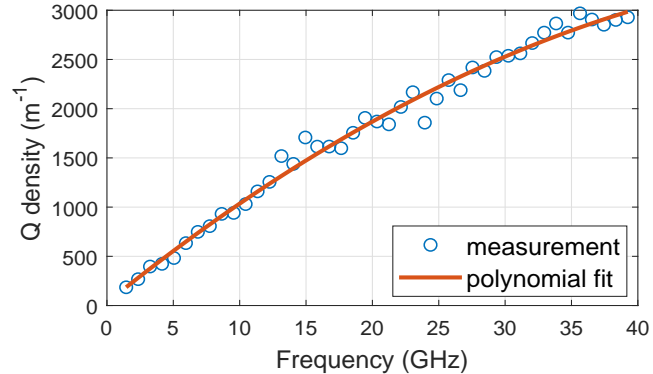

Fig. 2. Experimental estimate of $\mathrm{Q}$ density and the proposed model as a function of the center frequencies with $900 \mathrm{MHz}$ bandwidth

PDPs over all the Tx positions [6]. Hence, The averaged PDP (APDP) used for the RT estimation is calculated as

$$
A P D P=\sum_{p=1}^{14} \sum_{t x=1}^{10} \sum_{r x=1}^{36}|\operatorname{IDFT}\{H[f]\}|^{2}
$$

where IDFT $\{$.$\} is the inverse discrete Fourier transform, H[f]$ is the measured channel transfer funcion, and $r x, t x$ and $p$ are the indexes for the Rx antenna element, Tx antenna element and $\mathrm{Tx}$ position, respectively.

The slope of the APDP is determined automatically by fitting a least-square regression line to the values of the APDP within a certain delay interval. The fitting interval was chosen between the mean excess delay and the delay corresponding to $3 \mathrm{~dB}$ above the noise level, in order to minimize the influence of the LOS and noise components on the slope estimate [7]. The RT can then be experimentally determined as [6]

$$
\tau=\frac{-10 \log (e)}{\text { slope }}
$$

\section{RESULTS}

\section{A. $Q_{d}$ and RT Frequency-dependent Models}

The frequency range of $1-40 \mathrm{GHz}$ is divided into sub-bands of $900 \mathrm{MHz}$ bandwidth each, where the RT is estimated at each center frequency using (11). The estimated values are then used in (9) to obtain the $Q_{d}$, where $V=160 \mathrm{~m}^{3}$ and $A=195 \mathrm{~m}^{2}$ in our scenario. Fig. 2 presents the experimental $Q_{d}$ and the proposed fitting model as a function of frequency. The model for frequencies below $10 \mathrm{GHz}$ in [12] was chosen as a cubic polynomial fit, motivated by the fact that $Q$ is a cubic function of frequency. However, our measurements suggest that a simpler quadratic model can be a good fit for frequencies up to $40 \mathrm{GHz}$.

The dependence of the RT, and thus $Q_{d}$, on the bandwidth was discussed in [12]. It showed insignificant relative variation over bandwidths from $100 \mathrm{MHz}$ till $900 \mathrm{MHz}$ and concluded that $\tau$ can be considered constant over bands up to $900 \mathrm{MHz}$ or more. Similar behavior is observed in our scenario, and hence, a bandwidth of $900 \mathrm{MHz}$ is considered for the rest of the study, which provides enough samples for the APDP slope regression as well as the $Q_{d}$ model fitting. Therefore, the model of $Q_{d}$ is formulated as

$$
Q_{d}(f)=-0.86 f^{2}+109.25 f+29.49
$$




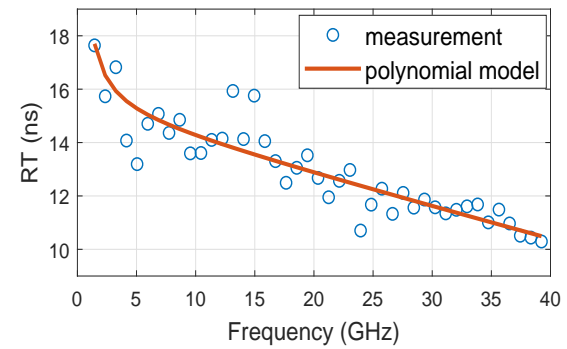

(a)

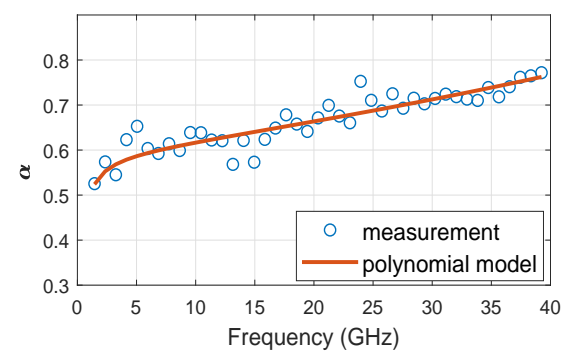

(b)

Fig. 3. Frequency-dependent models for (a) reverberation time and (b) average absorption coefficient and the experimental estimates with $900 \mathrm{MHz}$ bandwidth

where $f$ is the frequency in $\mathrm{GHz}$ and the goodness of fit in terms of the coefficient of determination $\left(\mathrm{R}^{2}\right)$ equals 0.99 . By substituting in (9), the frequency-dependent RT model is obtained

$$
\tau(f)=\frac{\left(-0.86 f^{2}+109.25 f+29.49\right) V}{2 \pi f A}[n s]
$$

The RT model is depicted in Fig. 3 (a) where it clearly shows that the RT is inversely proportional to the frequency. To validate our model, we compare the RT values to results reported in the literature. Since the RT also depends on the absorption rate of the environment, we consider the studies where the environment is similar to our scenario. However, a limited number of studies provide the dimensions of the indoor scenario, and most of them are at lower frequencies. Table I summaries the collected data and the relative error (RE) to the proposed model, where small deviations have been obtained.

\section{B. Average Absorption Coefficient}

Most of the results for the average absorption coefficient reported in the literature are based on the models of Sabine and Eyring [6] where the assumption of a constant wall interaction rate is adopted. The modified RT in (7) takes the variance of the number of interactions into account by relating it to $\gamma^{2}$. The later can only be calculated analytically for a limited number of room shapes [4], while other shapes can be determined by computer simulation. Hence, for our scenario, $\gamma^{2}$ is computed via ray-tracing simulation and is found to be 0.325 . This matches well with the results in [4] indicating that, for most rectangular rooms, $\gamma^{2}$ is close to 0.4 .

The frequency-dependent model of the average absorption coefficient $\alpha(f)$ can be easily determined from (7) and (13) and is shown in Fig. 3 (b) along with the experimental
TABLE I

RT Model VALidATION From THe Literature

\begin{tabular}{cccccc}
\hline Ref. & $\mathrm{V}\left(\mathrm{m}^{3}\right)$ & $\mathrm{A}\left(\mathrm{m}^{2}\right)$ & Freq. $(\mathrm{GHz})$ & $\mathrm{RT}(\mathrm{ns})$ & $\mathrm{RE}(\%)$ \\
\hline$[15]$ & 335.6 & 299.6 & 1.5 & 21.8 & 4.2 \\
{$[12]$} & 94.5 & 132 & $7.5-8.5-9.5$ & $14.3-13-12.2$ & 9.5 \\
{$[12]$} & 169 & 195 & $8-9-10$ & $14.2-12.1-13$ & 9.3 \\
\hline
\end{tabular}

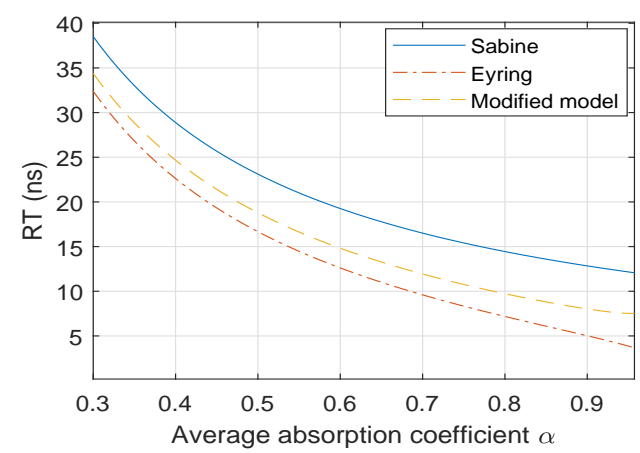

Fig. 4. Overview of reverberation models performance for our scenario

values. To show the improvement gained using the modified model, Fig. 4 plots the RT calculated based on (7) versus $\alpha$ and compares it to the models of Sabine and Eyring for our scenario. It shows that the modified RT is generally smaller than that obtained from Sabine's model, but larger than Eyring's.

The reason for the difference between the models is the underlying assumptions. Sabine assumes a steady-state uniform isotropic diffuse field (i.e. one energy value everywhere), which is not valid for high absorption. During energy decay, the room is not in steady-state, and the higher the absorption, the less it is in steady-state [4]. This results in a smaller effective energy loss and, thus, larger RT. On the other hand, Eyring assumes a constant rate of wall interactions and a stepwise energy decay (i.e. all rays lose energy at the same time) as shown in (1). This is valid for a 1-dimensional enclosure, where all paths have exactly the same length [4]. For a more accurate RT, we modified the model to take the variance of wall interactions into account. As the variance increases, the energy decay transitions from the step-wise assumption of Eyring to the continuous assumption of Sabine, and hence, the RT increases.

\section{CONClusion}

The frequency-dependency of the electromagnetic Q-factor and reverberation time is experimentally investigated in an indoor scenario from 1 to $40 \mathrm{GHz}$. The results demonstrate that the reverberation time decreases smoothly as the frequency increases, indicating that the higher the frequency, the faster the fading of diffuse fields. Models that predict the Q-factor and reverberation time are presented based on the theory of electromagnetic fields in cavities. These models extend the results found in the literature to new higher frequencies up to $40 \mathrm{GHz}$. Moreover, a model that predicts the average absorption coefficient from the reverberation time is proposed, which is theoretically more accurate than the commonly used models. 


\section{REFERENCES}

[1] European Project ICT-317669-METIS; Deliverable D8.4 METIS final project report, Tech. Rep., 2015.

[2] International Telecommunication Union (ITU); Final Acts WRC-15, World Radiocommunication Conference, Geneva, Tech. Rep., 2015.

[3] J. Poutanen, J. Salmi, K. Haneda, V.-M. Kolmonen, and P. Vainikainen, "Angular and shadowing characteristics of dense multipath components in indoor radio channels," IEEE Transactions on Antennas and Propagation, vol. 59, no. 1, pp. 245-253, 2011.

[4] H. Kuttruff, Room acoustics. Crc Press, 2016.

[5] J. B. Andersen, J. Nielsen, G. Pedersen, G. Bauch, and J. Herdin, "Room electromagnetics," IEEE Antennas and Propagation Magazine, vol. 49, no. 2, pp. 27-33, 2007.

[6] G. Steinböck, T. Pedersen, B. H. Fleury, W. Wang, and R. Raulefs, "Experimental validation of the reverberation effect in room electromagnetics," IEEE Transactions on Antennas and Propagation, vol. 63, no. 5, pp. 2041-2053, 2015.

[7] A. Bamba, W. Joseph, J. B. Andersen, E. Tanghe, G. Vermeeren, D. Plets, J. Ø. Nielsen, and L. Martens, "Experimental assessment of specific absorption rate using room electromagnetics," IEEE Transactions on Electromagnetic Compatibility, vol. 54, no. 4, pp. 747-757, 2012.

[8] D. A. Hill, Electromagnetic fields in cavities: deterministic and statistical theories. John Wiley \& Sons, 2009, vol. 35.

[9] W. C. Sabine and M. D. Egan, "Collected papers on acoustics," 1994.

[10] C. F. Eyring, "Reverberation time in dead rooms," The Journal of the Acoustical Society of America, vol. 1, no. 2A, pp. 217-241, 1930.

[11] O. Franek, J. B. Andersen, and G. F. Pedersen, "Diffuse scattering model of indoor wideband propagation," IEEE Transactions on Antennas and Propagation, vol. 59, no. 8, pp. 3006-3012, 2011.

[12] A. Bamba, M.-T. Martinez-Ingles, D. P. Gaillot, E. Tanghe, B. Hanssens, J.-M. Molina-Garcia-Pardo, M. Lienard, L. Martens, and W. Joseph, "Experimental investigation of electromagnetic reverberation characteristics as a function of uwb frequencies," IEEE Antennas and Wireless Propagation Letters, vol. 14, pp. 859-862, 2015.

[13] B. Hanssens, M.-T. Martínez-Inglés, E. Tanghe, D. Plets, J.-M. MolinaGarcía-Pardo, C. Oestges, L. Martens, and W. Joseph, "Measurementbased analysis of specular and dense multipath components at $94 \mathrm{ghz}$ in an indoor environment," IET Microwaves, Antennas \& Propagation, vol. 12, no. 4, pp. 509-515, 2017.

[14] M. Martinez-Ingles, J. Pascual-Garca, D. P. Gaillot, C. S. Borras, and J. Molina-Garca-Pardo, "Indoor 1-40 GHz channel measurements," in 2019 13th European Conference on Antennas and Propagation (EuCAP), March 2019, pp. 1-5.

[15] C. L. Holloway, M. G. Cotton, and P. McKenna, "A model for predicting the power delay profile characteristics inside a room," IEEE transactions on vehicular technology, vol. 48, no. 4, pp. 1110-1120, 1999. 